\title{
Potential for rapid antibody detection to identify tuberculous cattle with non- reactive tuberculin skin test results
}

\author{
W. Ray Waters ${ }^{1} \mathbb{D}$, H. Martin Vordermeier ${ }^{2}$, Shelley Rhodes², Bhagwati Khatri², Mitchell V. Palmer ${ }^{1}$, \\ Mayara F. Maggioli', Tyler C. Thacker', Jeffrey T. Nelson³, Bruce V. Thomsen³, Suelee Robbe-Austerman ${ }^{3}$, \\ Doris M. Bravo Garcia ${ }^{3}$, Mark A. Schoenbaum ${ }^{4}$, Mark S. Camacho ${ }^{5}$, Jean S. Ray ${ }^{6}$, Javan Esfandiarii, Paul Lambotte ${ }^{7}$, \\ Rena Greenwald ${ }^{7}$, Adrian Grandison ${ }^{7}$, Alina Sikar-Gang ${ }^{7}$ and Konstantin P. Lyashchenko ${ }^{7^{*}}$
}

\begin{abstract}
Background: Bovine tuberculosis (TB) control programs generally rely on the tuberculin skin test (TST) for ante-mortem detection of Mycobacterium bovis-infected cattle.

Results: Present findings demonstrate that a rapid antibody test based on Dual-Path Platform (DPP ) technology, when applied 1-3 weeks after TST, detected 9 of 11 and 34 of 52 TST non-reactive yet M. bovis-infected cattle from the US and GB, respectively. The specificity of the assay ranged from $98.9 \%(n=92, \mathrm{US})$ to $96.0 \%(n=50, \mathrm{~GB})$ with samples from TB-free herds. Multi-antigen print immunoassay (MAPIA) revealed the presence of antibodies to multiple antigens of $M$. bovis in sera from TST non-reactors diagnosed with TB.
\end{abstract}

Conclusions: Thus, use of serologic assays in series with TST can identify a significant number of TST non-reactive tuberculous cattle for more efficient removal from TB-affected herds.

Keywords: Antibody, Bovine tuberculosis, Dual path platform, Multi-antigen print immunoassay, Tuberculin skin test, Mycobacterium bovis

\section{Background}

Tuberculosis (TB) in humans and animals may result from exposure to bacilli within the Mycobacterium tuberculosis complex such as $M . t b$, M. bovis, M. africanum, M. microti, M. caprae, M. orygis, M. suricattae, $M$. mungi, or $M$. canetti $[1,2]$. M. bovis is the species most often isolated from tuberculous cattle. Despite intensive and costly control efforts over many decades, bovine TB persists in most countries adversely affecting animal health, welfare, and trade as well as the livelihoods of producers. Persistence of bovine TB in livestock populations also demands the maintenance of costly federal and regional regulatory networks. Control strategies rely largely on ante-mortem testing and slaughter inspection to identify livestock herds at risk. With cattle, the principal ante-mortem tests for

\footnotetext{
* Correspondence: KLyashchenko@chembio.com

${ }^{7}$ Chembio Diagnostic Systems, Inc., Medford, NY, USA

Full list of author information is available at the end of the article
}

presumptive diagnosis of bovine TB are immunoassays that detect cell-mediated responses, including both in vivo [i.e., tuberculin skin test (TST)] and in vitro [i.e., interferon gamma release assay (IGRA)] methods [3-5]. In many countries, TST is applied as the primary test and IGRA may be used as an ancillary test in cattle to maximize the number of infected animals identified or as a confirmatory test [6]. The most common applications for ante-mortem testing include routine surveillance to identify $M$. bovis-infected herds, test and removal schemes, movement tests, epidemiologic trace-back testing, and in TBaffected herds to delineate animals going to a slaughter plant versus being condemned for rendering. While used extensively for over 100 years in cattle, the TST does have a number of severe shortcomings. The sensitivity of TST ranges broadly from 55 to $97 \%$ depending on the type and technical variations of test applied, quality of purified protein derivative (PPD), environmental exposure/burden 
to atypical mycobacteria, and many other factors $[4,5]$. Thus, improved ante-mortem tests and/or testing strategies for bovine TB are greatly needed.

Over the past decade, a new generation of serologic tests designed to detect antibodies to multiple $M$. bovis specific antigens have emerged for application in cattle [7-13]. Of these, an ELISA using MPB83 and MPB70 antigens (M. bovis Ab Test, IDEXX Laboratories, Westbrook, Maine, US [10]) is approved by the Office International des Epizooties and US Department of Agriculture for discretionary use in cattle; however, application of this test has been limited primarily to confirmation of infection. Injection of PPDs for TST significantly boosts antibody responses in $M$. bovis infected cattle, including animals without prior detectable antibody responses [10,11, 14-16]. The enhanced IgG responses are elicited by $M$. bovis specific antigens (e.g., MPB83 and MPB70) and characterized by accelerated antibody affinity maturation [17]. The boosted antibody responses wane beginning $\sim 1-2$ months after PPD administration; however, they can be further increased upon PPD re-injection [17]. Despite these advances, existing antibody assays generally lack diagnostic sensitivity, especially in early infection, and thus require further improvements [4-6].

In the present study, sera from M. bovis-infected cattle identified as TST non-reactors in naturally-exposed herds within the US $(n=11)$ and GB $(n=52)$ were evaluated for antibody responses to $M$. bovis specific proteins using a next generation immunochromatographic test based on Dual-Path Platform (DPP) technology developed by Chembio Diagnostic Systems, Inc. (Medford, New York, US) [9]. Findings demonstrate the potential for use of antibody assays to detect $M$. bovis infection in TST non-reactive cattle within TB-affected herds.

\section{Methods \\ Naturally-infected herds \\ Great Britain}

Sera $(n=127)$ from GB were obtained from M. bovis-infected cattle detected during routine surveillance, including multiple herds and animals of diverse age, gender, breed, and management systems. All animals received a single intradermal comparative cervical test (SICCT) and of these, 52 animals were SICCT negative, IGRA positive (blood collected for IGRA and serum $\sim 60$ days postSICCT) with tuberculous lesions detected postmortem. The other 75 animals were SICCT positive and not tested by IGRA (serum collection circa 3 weeks post-SICCT at the abattoir) with tuberculous lesions upon postmortem and $M$. bovis isolated from lesions. Serum samples were also collected from 50 SICCT negative, IGRA negative cattle located in a TB-free region of GB.

\section{Texas, US}

A cow with tuberculous lesions (later confirmed as $M$. bovis upon mycobacterial culture) was detected upon routine inspection at an abattoir in 2014. The source herd of this cow was determined to consist of approximately 11,000 Holstein dairy cattle. On postmortem examination of initial caudal-fold tuberculin test (CFT) reactors, a tuberculous lesion rate (confirmed by histopathology) of $1.5 \%$ was found. Based on epidemiologic risk factors, it was determined by regulatory officials and dairy management that destocking the dairy would be the best approach to rid it of M. bovis. A CFT and collection of blood for serological testing was completed 10-15 days prior to postmortem examination at destocking. A $2 \%$ overall prevalence of TB (visible tuberculous lesions confirmed by histopathology) was noted in cattle among CFT positive (35\%) and CFT negative cattle (0.4\%). From the CFT false-negative cattle, 7 serum samples obtained within 3 weeks after PPD injection were available for serologic analysis.

\section{Michigan, US}

Two cattle herds, one beef and the other dairy (mostly Jersey), within the TB-endemic region of Michigan (Northeast corner of the lower peninsula) were identified as TB-affected via routine surveillance in 2015. Animals within these two herds had received CFTs yearly for TB surveillance prior to 2015. Upon identification of tuberculous animals in 2015, CFTs were applied more frequently ( 4-6 month interval) in both herds. Based on the presence of gross lesions upon depopulation, prevalence of TB was estimated to be $21 \%$ (17/81) and 9\% $(53 / 561)$ for the beef and dairy herds, respectively. Four serum samples collected from $M$. bovis-infected CFT non-reactors within 3 weeks after the last CFT administration were available for serologic testing.

\section{Multi-antigen print immunoassay (MAPIA)}

MAPIA was performed as described previously [18]. Briefly, a panel of ten $M$. tuberculosis-complex antigens immobilized on nitrocellulose membrane included ESAT-6 (Rv3875), CFP10 (Rv3874), MPB64 (Rv1980c), MPB70 (Rv2875), MPB83 (Rv2873), CFP10/ ESAT-6 fusion protein, MPB70/MPB83 fusion protein, MPB70/ CFP10/Rv0934 fusion protein, bovine PPD (bPPD), and M. bovis culture filtrate (MBCF). Strips were cut and blocked with $1 \%$ nonfat milk in PBS with $0.05 \%$ Tween 20 for $1 \mathrm{~h}$ prior to incubation with serum samples diluted 1:40 in blocking solution for $1 \mathrm{~h}$. After washing, strips were incubated with peroxidase-conjugated Protein G (Sigma, St. Louis, MO) diluted 1:1000 for $1 \mathrm{~h}$, washed, and developed with 3,3',5,5'-tetramethyl benzidine (TMB) (Kirkegaard \& Perry Laboratories, Inc., Gaithersburg, MD). 


\section{Dual-path platform (DPP ) assay}

Bovine IgG and IgM antibodies to CFP10/ESAT-6 and MPB70/MPB83 protein fusions were detected as described previously [17] using goat anti-bovine IgG and anti-bovine IgM antibodies (Kirkegaard \& Perry Laboratories Inc.) conjugated to colloidal gold nanoparticles by Chembio standard procedure. Sera were diluted 1:20 in sample buffer for testing by DPP assay and results were recorded at 15 min using an optical reader to measure test band reflectance in relative light units (RLU), as previously described [17]. Using pre-established cutoff values of 20 RLU for CFP10/ESAT- 6 antigen and 40 RLU for MPB70/MPB83 antigen (same for both IgM and IgG antibody detection), DPP assay readouts were expressed as signal-to-cutoff ratios, with any values $\geq 1.00$ being interpreted as a reactive result and any value $<1.00$ being considered as a non-reactive result.

\section{Data analysis}

Diagnostic performance of the serologic assays was assessed against the gold standard of $M$. bovis culture and/or IS-6110 PCR by calculating test sensitivity and specificity using available software [19] and presented with the 95\% confidence intervals (CI). Fisher's exact test was used for analysis of antigen recognition by bovine antibodies in the present study.

\section{Results}

Antibody detection in TST non-reactors versus TST reactors: Samples from Great Britain

With sera from $M$. bovis infected cattle in GB, the overall IgG reactivity rates in the DPP assay were similar for SICCT reactors $(60 \%, n=75)$ and non-reactors $(65.4 \%$, $n=52$ ). The DPP assay specificity assessed with sera from 50 SICCT negative, IGRA negative cattle from TB non-endemic regions of GB was 96\% (Table 1). Of note, the response rate by $M$. bovis-infected cattle to each fusion antigen differed significantly $(p<0.01$, Fisher's exact test) based on TST reactivity. The ratio of MPB70/ MPB83 to CFP10/ESAT-6 reactivity was approximately 3.6:1 for TST reactors versus 1:1 for TST non-reactors (Table 1). In line with the above, only 1 of 75 TST reactors but 13 of 52 TST non-reactors produced antibody solely to CFP10/ESAT-6 yet not to MPB70/ MPB83. Thus, the immunodominance of serologically related MPB70 and/or MPB83 proteins typically detected in M. bovis infected cattle after PPD administration for SICCT was less evident with TST non-reactors, as reactivity rates to CFP10/ESAT- 6 and MPB70/MPB83 were essentially equivalent, and there were considerably more CFP10/ESAT-6 antibody responders within the TST non-reactor subset. This observation demonstrates the potential value for use of additional antigens to maximize the sensitivity of serologic tests, particularly with TST non-reactors.

\section{Antibody detection in TST non-reactors: Opportunistic samples from the United States}

During 2015 - 2016, two herds from disparate regions of the US were under-going whole herd depopulation due to M. bovis infection within the herds. Antemortem testing was used to delineate animals going to a slaughter plant (test negative) versus being condemned for rendering (test positive). Serum samples were available for serologic analysis from 11 CFT non-reactive adult cows with gross tuberculous lesions from the two herds [Texas $(n=7)$ and Michigan $(n=4)]$. Infection was confirmed in these animals by histopathology, mycobacterial culture, and/or IS-6110 PCR (Table 2). Each of the animals had received a CFT $\sim 6$ months prior to the nonreactive CFT; of which 4/7 (Texas) and 0/4 (Michigan) were reactive at that earlier time point, thus indicating TST reversion in four of the animals. Serum samples collected 1-3 weeks after the last CFT were tested with the DPP assay for the presence of IgM and IgG antibodies to CFP10/ESAT-6 and MPB70/MPB83. Only 4/ 11 animals had IgM to MPB70/MPB83, whereas none produced IgM to CFP10/ESAT-6. In contrast, relatively potent IgG responses were elicited by MPB70/MPB83 and CFP10/ESAT- 6 in 8/11 and 3/11 animals, respectively (Table 3 ). IgM readouts were generally lower than those obtained for IgG, suggesting little added value of IgM antibody detection from a serodiagnostic sensitivity perspective. The one exception was with animal \#364 which had a high IgM and a borderline

Table $1 \mathrm{lgG}$ reactivity rates in tuberculin skin test reactors and non-reactors found among M. bovis-infected cattle in GB

\begin{tabular}{|c|c|c|c|c|}
\hline \multirow[t]{2}{*}{ Animal group ${ }^{a}$} & \multirow[t]{2}{*}{ No. of animals } & \multicolumn{2}{|c|}{ Individual antigen reactivity rates in DPP assay ${ }^{b}$} & \multirow{2}{*}{$\begin{array}{l}\text { DPP assay reactivity rate }{ }^{b} \\
(\%, 95 \% \mathrm{Cl})\end{array}$} \\
\hline & & CFP10/ESAT-6 & MPB70/MPB83 & \\
\hline M. bovis infected, TST Reactors & 75 & $12(16 \%)$ & $43(57.3 \%)$ & 45 (60\%; 95\% Cl: 48,71.2) \\
\hline M. bovis infected, TST Non-Reactors & 52 & $21(40.4 \%)$ & $21(40.4 \%)$ & 34 (65.4\%, 95\% Cl: 50.9, 78) \\
\hline Total M. bovis infected & 127 & $33(26 \%)$ & $64(50.4 \%)$ & 79 (62.2\%, 95\% Cl: 53.2, 70.6) \\
\hline TB-free, TST Non-Reactors & 50 & $2(4 \%)$ & $2(4 \%)$ & $2(4 \%, 95 \%$ Cl: $0.5,13.7)$ \\
\hline
\end{tabular}

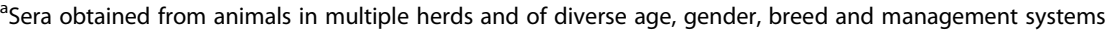

${ }^{\mathrm{b}}$ Data are presented as number (percent) positive per group for each antigen or assay 
Table 2 Diagnostic characterization of TST false-negative cattle identified in US herds infected with M. bovis

\begin{tabular}{|c|c|c|c|c|c|c|c|}
\hline \multirow[t]{2}{*}{ State } & \multirow[t]{2}{*}{ Animal ID \# } & \multicolumn{2}{|c|}{ CFT' results } & \multicolumn{4}{|c|}{ Postmortem examination results } \\
\hline & & Jul-2015 & Jan-2016 & Gross lesions & Histopathology & $P C R^{b}$ & $\overline{\text { Culture }}$ \\
\hline \multirow[t]{7}{*}{$\overline{T X}$} & 755 & Pos & Neg & Present & TB compatiblec, IS6110 Pos ${ }^{d}$ & Neg & Neg \\
\hline & 272 & Pos & Neg & Present & TB compatible & Pos & $N D^{e}$ \\
\hline & 976 & Neg & Neg & Present & TB compatible & Pos & ND \\
\hline & 857 & Pos & Neg & Present & TB compatible & Pos & ND \\
\hline & 676 & Pos & Neg & Present & TB compatible & Pos & ND \\
\hline & 889 & Pos & Neg & Present & TB compatible & Pos & ND \\
\hline & 352 & Neg & Neg & Present & TB compatible & Pos & ND \\
\hline \multirow[t]{6}{*}{$\mathrm{MI}^{\mathrm{f}}$} & & Sep-2015 & Jan-2016 & & & & \\
\hline & 855 & Neg & Neg & Present & TB compatible & ND & M. bovis \\
\hline & 370 & Neg & Neg & Present & TB compatible & ND & M. bovis \\
\hline & 364 & Neg & Neg & Present & Not compatible & ND & M. bovis \\
\hline & & May-2015 & Oct-2015 & & & & \\
\hline & 809 & Neg & Neg & Present & TB compatible & ND & M. bovis \\
\hline
\end{tabular}

${ }^{a}$ CFT, caudal fold test (i.e., intradermal M. bovis PPD injected into the caudal skin fold and response determined by palpation $72 \mathrm{~h}$ after injection) was applied $<3$ wks prior to collection of serum

${ }^{\mathrm{b}} \mathrm{PCR}$, polymerase chain reaction for either $M$. tb complex IS6110 or 1081 DNA on fresh tissue

${ }^{c}$ Microscopic granulomatous lesions consistent with bovine TB and containing acid-fast bacilli

${ }^{\mathrm{d}}$ IS6110 DNA by polymerase chain reaction on formalin-fixed tissue

ND, not done

${ }^{f}$ Animals \# 855, 370, and 364 were from a beef herd while \#809 was from a dairy herd

IgG signal (Table 3). Development of transient IgM responses early in the course of experimental $M$. bovis infection, as well as shortly after TST administration, has been demonstrated in previous studies using different strains of $M$. bovis [11, 17, 20]. Thus, the present findings in the US demonstrated antibody responses to $M$. bovis antigens in $\sim 82 \%$ of TST nonreactive cattle with confirmed $\mathrm{TB}$. The specificity of

Table 3 Quantitative measure of IgM and IgG responses produced by TST false-negative cattle in the US

\begin{tabular}{|c|c|c|c|c|}
\hline \multirow{3}{*}{$\begin{array}{l}\text { Animal } \\
\text { ID \# }\end{array}$} & \multicolumn{4}{|c|}{ Reactivity in the DPP assay $^{a}$} \\
\hline & \multicolumn{2}{|c|}{ CFP10/ESAT-6 } & \multicolumn{2}{|c|}{ MPB70/MPB83 } \\
\hline & $\lg M$ & $\lg G$ & $\lg M$ & $\lg G$ \\
\hline 755 & 0 & 0.35 & 0 & 6.15 \\
\hline 272 & 0 & 0 & 1.15 & 1.08 \\
\hline 976 & 0 & 1.50 & 0.75 & 7.45 \\
\hline 857 & 0 & 0 & 0.15 & 0 \\
\hline 676 & 0 & 0 & 1.48 & 4.85 \\
\hline 889 & 0 & 2.05 & 0 & 19.15 \\
\hline 352 & 0 & 0.30 & 0 & 5.00 \\
\hline 855 & 0 & 0 & 0 & 0 \\
\hline 370 & 0 & 0 & 3.10 & 14.95 \\
\hline 364 & 0 & 0.50 & 6.78 & 1.58 \\
\hline 809 & 0 & 14.45 & 0.55 & 6.23 \\
\hline
\end{tabular}

${ }^{a}$ Data are presented as signal-to-cutoff ratios with $\geq 1.00$ considered as antibody reactive results (shown in bold) the DPP assay evaluated on 92 samples from TB-free states within the US was $98.9 \%$ (95\% CI: 94.1, 99.9) or somewhat higher than that found in GB $(96.0 \%$; 95\% CI: 86.3, 99.5), although not statistically significant $(p=0.3)$.

\section{Antigen recognition by lgG antibodies in sera from TST non-responders}

Serum samples from the US cattle were also analyzed by MAPIA to determine antigen recognition patterns. Sera from 10 of 11 cattle reacted with multiple recombinant antigens of $M$. bovis (Fig. 1). The one animal (\#855) which had a negative result in the DPP assay, exhibited IgG binding only to MBCF in MAPIA. Animal \#857, which was the second DPP non-reactor in this group (Table 3), displayed in MAPIA weak reactivity with the two fusion antigens containing MPB70. Based on line intensity, the magnitude of antibody responses and antigen recognition profiles varied among the animals. The most reactive antigens included MPB70 and MPB83 proteins, MPB70/MPB83 and MPB70/CFP10/Rv0934 hybrids, as well as bPPD and MBCF. For M. bovis-infected TST non-reactors, the role of CFP10/ESAT-6 in eliciting antibody responses was not significant in the US (Table 3, Fig. 1) as compared to the GB set of specimens (Table 1). Overall, the antigen recognition results supported the immunodominance of MPB70 and MPB83 proteins in $M$. bovis infection of cattle $[8,11,17,20]$. 


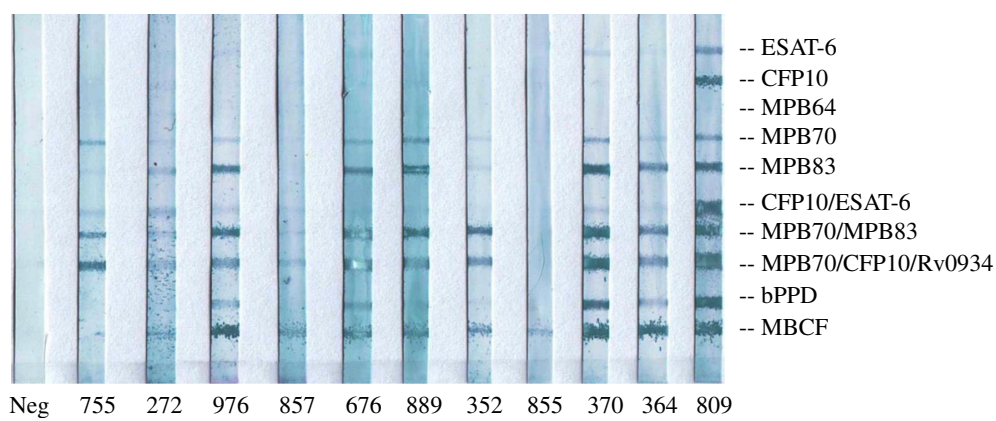

Fig. 1 MAPIA testing of TST non-reactive cattle diagnosed with M. bovis infection in the US. Assay was performed as described in Methods. Antigens printed onto nitrocellulose membrane are shown on the right. Results are presented for sera from a negative control (on the left), 7 animals from TX, and 4 animals from MI. Animal ID numbers are shown on the bottom (see Table 2 for diagnostic characterization). Visible bands on the strips indicate the presence of $\lg G$ to corresponding antigen(s). Intensity of the bands generally correlates with the antibody level

\section{Discussion}

A major impediment to the control of bovine TB is the relatively poor accuracy of current ante-mortem tests compounded by difficulties in reliably detecting tuberculous lesions and/or the agent in all infected animals upon slaughter surveillance. TST non-reactive cattle are particularly problematic when applying test and remove strategies in TB-affected herds. According to Lepper et al. [21], "Anergy to tuberculin is defined as the failure of an animal with visible evidence of tuberculosis to show a palpable cutaneous delayed hypersensitivity response to a tuberculin, at the time when the test is read." Such TST non-reactive animals, however, may still be responsive on other cell-mediated (e.g., IGRAs [22, 23]) or antibody detection immunoassays [24, 25]. In general, TST non-responsiveness is more common in animals from herds with high within-herd prevalence and in animals at advanced stages of disease [21, 22]. Desensitization as a result of repeated short interval application of TSTs may also lead to reduced TST responses associated with increased interleukin-10 (IL-10) and decreased IL-1 $\beta$ production to TB antigens [26]. Our findings further demonstrate that certain $M$. bovis-infected cattle can escape detection by TST by reverting from reactors to nonreactors within several months; however, a significant proportion of these animals can be identified by ancillary tests, such as the antibody tests described herein or IGRA [27].

In the present study, M. bovis specific antibody was detected by the DPP assay in $\sim 82 \%$ and $\sim 65 \%$ of $M$. bovis-infected, TST non-reactive cattle from the US and GB, respectively. As reported previously, an in-house MPB83-based ELISA detected 9 of 20 (45\%) SICCT non-reactors diagnosed with bovine TB in GB [28]. Similarly, the MPB70/83 antibody reactivity rate in the group of TST non-reactive cattle in GB was $\sim 40 \%$ as demonstrated by DPP assay (for MPB70/MPB83 antigen test line only, present study) and also shown independently by a commercial ELISA (M. bovis Ab Test, IDEXX Laboratories, Westbrook, Maine) using a cocktail of MPB70 and MPB83 proteins (unpublished data). Importantly, integrating CFP10/ESAT-6 to supplement MPB70/ MPB83 antigen in the DPP assay enhanced the overall test sensitivity to $\sim 65 \%$ in TST non-reactive cattle in GB, thereby highlighting the added benefit of combining multiple antigens in serologic assays.

\section{Conclusions}

The present findings demonstrate the potential for use of antibody tests in TB-affected herds to rapidly identify $M$. bovis-infected, but TST non-reactive cattle. The DPP assay may also be considered for use in series with TST as a movement test, particularly as the assay may be applied pen-side without the need for laboratory equipment and results are available within $20 \mathrm{~min}$. For example, Mexican cattle are required to have a negative CFT within 60 days of entry into the US. Thus, the DPP assay could be applied after CFT in Mexico or at the US/Mexico border as a further safeguard against entry of $M$. bovis-infected cattle into the US. For this application, given a very low disease prevalence in the large number of cattle crossing the US border with recent CFT negative results, a viable serologic assay would need to have an extremely high specificity (>99.9\%) to provide an acceptable positive predictive value at a low pre-test probability. In the case of the DPP assay, the target specificity can be established and validated by having a cut-off value adjusted to meet this key requirement without a significant loss of diagnostic sensitivity. Given the limited number of samples available for the study, present findings should be considered preliminary and more extensive studies particularly in other bovine TB endemic countries are warranted to further verify the utility of this approach. These studies should also include sera from cattle infected with non-tuberculous mycobacteria (e.g., M. avium subsp. paratuberculosis, $M$. 
kansasii, etc.) to determine the possible interaction of these mycobacteria on this approach, particularly as injection of PPDs for SICCT in cattle vaccinated with heat inactivated Johne's disease vaccine may induce false positive responses to MPB83/70-based antibody assays [29]. With that said, present findings clearly demonstrate that use of serologic assays in series with TST can identify a significant number of TST non-reactive tuberculous cattle for more efficient removal from TB-affected herds.

\begin{abstract}
Abbreviations
CFP10: Culture filtrate protein $10 \mathrm{kDa}$ protein; CFT: Caudal fold test; Cl: Confidence interval; DPP: Dual path platform; ESAT-6: Early secretory antigenic target 6 kDa protein; GB: Great Britain; IGRA: Interferon- $\gamma$ release assay; MAPIA: Multi-antigen print immunoassay; MPB70: Mobility protein of bovis, 70; MPB83: Mobility protein of bovis, 83; PPD: Purified protein derivative; RLU: Relative light units; SICCT: Single intradermal comparative cervical test; TB: Tuberculosis; TST: Tuberculin skin test; US: United States
\end{abstract}

\section{Acknowledgements}

We thank the Texas Animal Health Commission and Michigan Department of Agriculture for procurement of samples and providing supportive data. We thank Allison Lasley from USDA-ARS and Kent Munden from USDA-APHIS-VS for excellent technical support.

\section{Availability of data and materials}

The datasets supporting the conclusions of this article are included within the article.

\section{Funding}

This work was partially supported by the USDA, National Institute of Food and Agriculture, Small Business Innovative Research [Award \#2015-3361023505, \#2016-33610-25688], and the Department for Environment, Food and Rural Affairs (Defra) UK including funding from the devolved authorities. HMV is a Jenner Investigator.

\section{Authors' contributions}

WRW and KPL conceived the studies and wrote the initial draft of the manuscript. All authors reviewed and provided input into the crafting of the manuscript. JE, PL, RG, AG, ASG, and KPL performed all DPP and MAPIA assays. WRW, TC, MM, MP, MS, MC, JN, and JR organized collection and distribution of serum samples and supportive data from the US. SR, BK and HMV organized collection of serum samples and supportive data from the UK. BT performed microscopic analysis of tissues from samples collected in the US. DMG and SRA provided mycobacterial culture and PCR data. All authors read and approved the final manuscript.

\section{Competing interests}

USDA is an equal opportunity provider and employer. Mention of trade names or commercial products in this publication is solely for the purpose of providing specific information and does not imply recommendation or endorsement by the U.S. Department of Agriculture. JE, PL, RG, AG, ASG, and KPL are employees of Chembio Diagnostic Systems, Inc.

\section{Consent for publication}

All authors have read and approved the manuscript for submission and publication. Administrators from each agency (i.e., USDA-ARS, USDA-APHIS, APHA, DEFRA) and Chembio have approved the manuscript for submission and publication.

\section{Ethics approval and consent to participate}

Serum samples, postmortem samples (i.e., tissues for gross and microscopic inspection and mycobacterial culture and P(R), and TST results were obtained in compliance with official guidelines for the control and eradication of bovine TB in the US [i.e., US Department of Agriculture (USDA), Animal and Plant Health Inspection Services (APHIS), Veterinary Services] and GB [i.e., Department for Environment, Food and Rural Affairs (DEFRA)].

\section{Publisher's Note}

Springer Nature remains neutral with regard to jurisdictional claims in published maps and institutional affiliations.

\section{Author details}

${ }^{1}$ National Animal Disease Center, Agricultural Research Service, United States Department of Agriculture (USDA), Ames, IA, USA. ${ }^{2}$ Tuberculosis Research Group, Animal and Plant Health Agency, Addlestone, UK. ${ }^{3}$ National Veterinary Services Laboratories, Animal and Plant Health Inspection Service (APHIS), USDA, Ames, IA, USA. ${ }^{4}$ Veterinary Services (VS), APHIS, USDA, Fort Collins, CO, USA. ${ }^{5}$ VS, APHIS, USDA, Raleigh, NC, USA. ${ }^{6}$ S, APHIS, USDA, East Lansing, MI, USA. ${ }^{7}$ Chembio Diagnostic Systems, Inc., Medford, NY, USA.

Received: 9 September 2016 Accepted: 31 May 2017

Published online: 07 June 2017

\section{References}

1. Alexander KA, Sanderson CE, Larsen MH, Robbe-Austerman S, Williams MC, Palmer MV. Emerging tuberculosis pathogen hijacks social communication behavior in the group-living banded mongoose (Mungos mungo). MBio. 2016;7:e00281-16.

2. Clark C, van Helden P, Miller M, Parsons S. Animal-adapted members of the Mycobacterium tuberculosis complex endemic to southern African subregion. J South African Vet Assn. 2016;87:a11322.

3. Buddle BM, de Lisle GW, Waters WR, Vordermeier HM. Diagnosis of Mycobacterium bovis infection in cattle, vol. 9. In: Mukundan $\mathrm{H}$, Chambers MA, Waters WR, Larsen MH, editors. Many Hosts of Mycobacteria: Tuberculosis, Leprosy, and other Mycobacterial Diseases of Man and Animals. Oxfordshire: CABI Publishing; 2015. p. 168-84.

4. de la Rua-Domenech R, Goodchild AT, Vordermeier HM, Hewinson RG, Christiansen KH, Clifton-Hadley RS. Ante mortem diagnosis of tuberculosis in cattle: a review of the tuberculin tests, gamma-interferon assay and other ancillary diagnostic techniques. Res Vet Sci. 2006;81:190-210.

5. Schiller I, Oesch B, Vordermeier HM, Palmer MV, Harris BN, Orloski KA, et al. Bovine tuberculosis: a review of current and emerging diagnostic techniques in view of their relevance for disease control and eradication. Transbound Emerg Dis. 2010;57:205-20.

6. Bezos J, Casal C, Romero B, Schroeder B, Hardegger R, Raeber AJ, et al. Current ante-mortem techniques for diagnosis of bovine tuberculosis. Res Vet Sci. 2014;97:S44-52.

7. Green LR, Jones CC, Sherwood AL, Garkavi IV, Cangelosi GA, Thacker TC, et al. Single-antigen serological testing for bovine tuberculosis. Clin Vaccine Immunol. 2009;16:1309-13.

8. Lyashchenko K, Whelan AO, Greenwald R, Pollock JM, Andersen P, Hewinson RG, et al. Association of tuberculin-boosted antibody responses with pathology and cell-mediated immunity in cattle vaccinated with Mycobacterium bovis BCG and infected with M. bovis. Infect Immun. 2004:72:2462-7.

9. Lyashchenko KP, Greenwald R, Esfandiari J, O'Brien DJ, Schmitt SM, Palmer MV, et al. Rapid detection of serum antibody by dual-path platform VetTB assay in white-tailed deer infected with Mycobacterium bovis. Clin Vaccine Immunol. 2013;20:907-11.

10. Waters WR, Buddle BM, Vordermeier HM, Gormley E, Palmer MV, Thacker TC, et al. Development and evaluation of an enzyme-linked immunosorbent assay for use in the detection of bovine tuberculosis in cattle. Clin Vaccine Immunol. 2011;18:1882-8.

11. Waters WR, Palmer MV, Thacker TC, Bannantine JP, Vordermeier HM, Hewinson RG, et al. Early antibody responses to experimental Mycobacterium bovis infection of cattle. Clin Vaccine Immunol. 2006;13:648-54.

12. Whelan C, Shuralev E, O'Keeffe G, Hyland P, Kwok HF, Snoddy P, et al. Multiplex immunoassay for serological diagnosis of Mycobacterium bovis infection in cattle. Clin Vaccine Immunol. 2008;15:1834-8.

13. Whelan C, Whelan AO, Shuralev E, Kwok HF, Hewinson G, Clarke J, et al. Performance of the Enferplex TB assay with cattle in great Britain and assessment of its suitability as a test to distinguish infected and vaccinated animals. Clin Vaccine Immunol. 2010;17:813-7.

14. Casal C, Díez-Guerrier A, Álvarez J, Rodriguez-Campos S, Mateos A, Linscott $R$, et al. Strategic use of serology for the diagnosis of bovine tuberculosis after intradermal skin testing. Vet Microbiol. 2014;170:342-51. 
15. Lightbody KA, Skuce RA, Neill SD, Pollock JM. Mycobacterial antigen-specific antibody responses in bovine tuberculosis: an ELISA with potential to confirm disease status. Vet Rec. 1998;142:295-300.

16. Lightbody KA, McNair J, Neill SD, Pollock JM. IgG isotype antibody responses to epitopes of the Mycobacterium bovis protein MPB70 in immunised and in tuberculin skin test-reactor cattle. Vet Microbiol. 2000;75:177-88

17. Waters WR, Palmer MV, Stafne MR, Bass KE, Maggioli MF, Thacker TC, et al. Effects of serial skin testing with purified protein derivative on the level and quality of antibodies to complex and defined antigens in Mycobacterium bovis-infected cattle. Clin Vaccine Immunol. 2015;22:641-9.

18. Lyashchenko KP, Singh M, Colangeli R, Gennaro ML. A multi-antigen print immunoassay for the development of serological diagnosis of infectious diseases. J Immunol Methods. 2000;242:91-100.

19. Lowry R. VassarStats: Website for Statistical Computation. Clinical Research Calculators. http://vassarstats.net/. Accessed 5 June 2017.

20. Waters WR, Thacker TC, Nelson JT, DiCarlo DM, Maggioli MF, Greenwald R, et al. Virulence of two strains of Mycobacterium bovis in cattle following aerosol infection. J Comp Pathol. 2014;151:410-9.

21. Lepper AW, Pearson CW, Corner LA. Anergy to tuberculin in beef cattle. Aust Vet J. 1977;53:214-6.

22. Houlihan MG, Dixon FW, Page NA. Outbreak of bovine tuberculosis featuring anergy to the skin test, udder lesions and milkborne disease in young calves. Vet Rec. 2008;163:357-61.

23. Sinclair JA, Dawson KL, Buddle BM. The effectiveness of parallel gammainterferon testing in New Zealand's bovine tuberculosis eradication programme. Prev Vet Med. 2016;127:94-9.

24. Lilenbaum W, Fonseca LS. The use of Elisa as a complementary tool for bovine tuberculosis control in Brazil. Braz J Vet Res Anim Sci. 2006;43:256-61.

25. Plackett P, Ripper J, Corner LA, Small K, de Witte K, Melville L, et al. An ELISA for the detection of anergic tuberculous cattle. Aust Vet J. 1989;66:15-9.

26. Coad M, Clifford D, Rhodes SG, Hewinson RG, Vordermeier HM, Whelan AO. Repeat tuberculin skin testing leads to desensitisation in naturally infected tuberculous cattle which is associated with elevated interleukin-10 and decreased interleukin-1 beta responses. Vet Res. 2010;41:14

27. Wood PR, Jones SL. BOVIGAM: an in vitro cellular diagnostic test for bovine tuberculosis. Tuberculosis (Edinb). 2001:81:147-55.

28. Coad M, Downs SH, Durr PA, Clifton-Hadley RS, Hewinson RG, Vordermeier HM, et al. Blood-based assays to detect Mycobacterium bovis-infected cattle missed by tuberculin skin testing. Vet Rec. 2008;162:382-4.

29. Coad M, Clifford DJ, Vordermeier HM, Whelan AO. The consequences of vaccination with the Johne's disease vaccine, Gudair, on diagnosis of bovine tuberculosis. Vet Rec. 2013;172(10):266

\section{Submit your next manuscript to BioMed Central and we will help you at every step:}

- We accept pre-submission inquiries

- Our selector tool helps you to find the most relevant journal

- We provide round the clock customer support

- Convenient online submission

- Thorough peer review

- Inclusion in PubMed and all major indexing services

- Maximum visibility for your research

Submit your manuscript at www.biomedcentral.com/submit 